\section{Handbook of Vinyl Polymers: Radical Polymerisation, Process and Technology}

by Munmaya K. Mishra and Yusuf Yagci: CRC Press, Boca Raton, FL, USA, 2nd ed., 2008, pp 784, ISBN-13:978-0-8247-2595-2.

Price: GBP 127.00, USD 199.95

This is a wide ranging book consisting of twenty-one chapters split into five sections. The first two sections cover the fundamentals and polymer initiating systems. The eleven chapters in these two sections are written by the editors alone for the first section and in collaboration with others for the second section. The later sections on "Technical Processes of Vinyl Polymerisation" and "Vinyl Polymer Technology" are by a range of authors. The final section, again written by the editors, is a useful compilation of factual data.

The fundamentals are covered briefly but adequately with few references. The second section covers the various polymerisation mechanisms in considerable detail. In addition to the detailed descriptions of all of the initiator systems for polymerisation, there are chapters specifically covering the involvement of initiators and the mechanisms concern- ing "Functionalization of Polymers", "Controlled/Living Radical Polymerisation" and "Block and Graft Copolymers". The chapters in these first two sections can be read in isolation but they clearly fit together well to cover the topics systematically.

The two chapters of the third section describe the various industrial polymerisation processes and how they are applied to the common polymer systems. There is inevitably some repetition of initiator mechanisms but this again means that the chapters can be read in isolation.

The third section of the book covers a range of topics and is rather more variable in scope and quality. The first chapter of this section covers "Vinyl Polymer Degradation". The first part of this chapter described polymer degradation generally but then covers a selection of vinyl polymers in some detail; however, there are a lot of common vinyl polymer types not covered. There is a section on "Characterisation of Polymer Degradation-Analytical Techniques" but the lack of mention of any chromatographic techniques (including GPC/ SEC) is a disappointment to a chromatographer. There are separate chapters on "Fiber-filled and particulate-filled vinyl polymer composites". The first of these chapters has an introduction with descriptions of some vinyl polymer types but the selection again excludes a lot of common vinyl polymer types. While there is useful discussion of the different types of fibers, the processes used and the interactions between polymer and fiber, this chapter did seem a bit disjointed. The corresponding chapter on particulate-filled composites appeared more logical and comprehensive in its coverage, with plenty of consideration being given to the developing areas of nanocomposites. The next two chapters in this section cover applications and recycling of vinyl polymers in a comprehensive manner. There is a chapter on processing of vinyl polymers which is good but really only describes PVC and PVC blends. The final chapter covers "Characterisation of Interfaces in Composites Using Micro-Mechanical Techniques" and while it does describe techniques likely to be of interest to those involved with composite materials, there is nothing specific to vinyl polymers.

This book is wide ranging and each of the chapters can be read in isolation. All of the main chapters have a good selection of references and there is a lot of useful information compiled in the final section. Some chapters do not really cover the breadth of vinyl polymers.

S. Holding 